\title{
Clinical and mycological predictors of cryptococcosis-associated immune reconstitution inflammatory syndrome
}

\author{
Christina C. Chang ${ }^{a, b, c, d}$, Afton A. Dorasamy ${ }^{e, f}$, Bernadett I. Gosnell ${ }^{b}$, \\ Julian H. Elliott ${ }^{a}$, Tim Spelman ${ }^{a, g}$, Saleha Omarjee ${ }^{c}$, \\ Vivek Naranbhai ${ }^{\mathrm{c}, \mathrm{h}}$, Yacoob Coovadia ${ }^{\mathrm{e}, \mathrm{f}}$, Thumbi Ndung' $\mathbf{u}^{\mathrm{c}}$, \\ Mohamed-Yunus S. Moosab , Sharon R. Lewin ${ }^{a, d}$ and Martyn A. French ${ }^{\mathrm{i}, j}$
}

\begin{abstract}
Objective: HIV-infected patients with treated cryptococcal meningitis are at risk for further neurological deterioration after commencing combination antiretroviral therapy (cART), mostly because of cryptococcosis-associated immune reconstitution inflammatory syndrome (C-IRIS). Identifying predictors of C-IRIS could enable risk stratification.
\end{abstract}

Design: Prospective, longitudinal cohort study for 24 weeks.

Setting: Durban, South Africa.

Participants: One hundred and thirty HIV-infected patients with first cryptococcal meningitis episode

Intervention: Antifungal therapy (amphotericin $1 \mathrm{mg} / \mathrm{kg}$ median 14 days, followed by consolidation and maintenance fluconazole) and CART (commenced median of 18 days from cryptococcal meningitis diagnosis).

Main outcome measure: Clinical, blood, and cerebrospinal fluid (CSF) markers associated with C-IRIS before and during CART and clinical significance of CSF cryptococcal culture negativity pre-cART commencement.

Results: Of 106 patients commencing CART, 27 (25.5\%) developed C-IRIS, 16 (15.1\%) neurological deterioration-not C-IRIS, and 63 (59.4\%) no neurological deterioration. On multivariable analysis, C-IRIS was associated with persistent CSF cryptococcal growth [hazard ratio (HR) $0.27, P=0.026$ ] and lower CSF protein (HR 0.53, $P=0.059$ ) prior to cART and lower $\mathrm{CD}^{+}{ }^{+} \mathrm{T}$-cell increases (HR 0.99, $\left.P=0.026\right)$ but not change in HIV viral load during cART. Using survival analysis, patients with a negative cryptococcal culture pre-cART commencement $(n=51 ; 48.1 \%$ ) experienced fewer episodes of neurological deterioration, C-IRIS, and cryptococcal relapse/persistence than patients with culture positivity $(n=55 ; 51.9 \%, \mathrm{HR} 0.33,0.33$, and 0.12 and $P=0.0003,0.0042$, and 0.0004 , respectively).

\footnotetext{
${ }^{a}$ Department of Infectious Diseases, Alfred Hospital and Monash University, Melbourne, Australia, ${ }^{b}$ Department of Infectious Diseases, King Edward VIII Hospital, ' HIV Pathogenesis Programme, Doris Duke Medical Research Institute, Nelson R Mandela School of Medicine, University of KwaZulu-Natal, Durban, South Africa, ${ }^{\mathrm{d} C e n t r e}$ for Virology, Burnet Institute, Melbourne, Australia, ${ }^{e}$ Department of Medical Microbiology, National Health Laboratory Services, Inkosi Albert Luthuli Central Hospital Academic Complex, ${ }^{\mathrm{f}}$ Department of Microbiology and Infection Control, School of Laboratory Medicine and Medical Sciences, University of KwaZulu-Natal, Durban, South Africa, ${ }^{\mathrm{g}} \mathrm{Centre}$ for Population Health, Burnet Institute, Melbourne, Australia, ${ }^{\mathrm{h}}$ Centre for the AIDS Programme of Research in South Africa, Doris Duke Medical Research Institute, Nelson R Mandela School of Medicine, University of KwaZulu-Natal, Durban, South Africa, 'School of Pathology and Laboratory Medicine, University of Western Australia, and 'Department of Clinical Immunology, Royal Perth Hospital and PathWest Laboratory Medicine, Perth, Australia.

Correspondence to Professor Martyn A. French, MD, Royal Perth Hospital, Perth, Western Australia, Australia.

Tel: +61 89224 2899; fax: +61 89224 2920; e-mail: Martyn.French@uwa.edu.au

Received: 18 January 2013; revised: 15 March 2013; accepted: 15 March 2013.
}

DOI:10.1097/QAD.0b013e3283614a8d

ISSN 0269-9370 @ 2013 Wolters Kluwer Health | Lippincott Williams \& Wilkins Copyright @ Lippincott Williams \& Wilkins. Unauthorized reproduction of this article is prohibited. 
Conclusion: Persistent CSF cryptococcal growth at cART initiation and poor CD4 ${ }^{+}$ T-cell increases on cART are strong predictors of C-IRIS. Approaches aimed at achieving CSF culture negativity prior to CART should be evaluated as a strategy to reduce rates of C-IRIS.

(C) 2013 Wolters Kluwer Health | Lippincott Williams \& Wilkins

AIDS 2013, 27:2089-2099

\section{Keywords: cryptococcal meningitis, cryptococcosis-associated immune reconstitution inflammatory syndrome, HIV/AIDS, immune restoration disease}

\section{Introduction}

Propelled by the HIV epidemic, cryptococcal meningitis is currently the leading cause of adult meningitis in central and southern Africa. Globally, two-thirds of the 1 million patients with cryptococcal meningitis are estimated to die within 3 months of diagnosis [1]. Patients with HIV infection who survive cryptococcal meningitis to commence combination antiretroviral therapy (cART) often experience further neurological deterioration presenting with worsening headaches, seizures, or confusion. This can lead to further invasive procedures and hospital admissions for empirical re-treatment of cryptococcal meningitis and is associated with significant morbidity and mortality. A large proportion of these neurological deterioration events are likely because of paradoxical cryptococcosis-associated immune reconstitution inflammatory syndrome (C-IRIS).

Paradoxical C-IRIS is a form of immune restoration disease, a condition resulting from heightened immune responses against viable and/or nonviable opportunistic pathogens that cause immunopathology at various sites [2] and has been reported in up to $50 \%$ of patients following commencement of cART [3]. Diagnosis of immune restoration disease requires careful exclusion of alternative diagnoses, a temporal association to cART commencement, and evidence of a virological response to cART [4-6]. Specific definitions for diagnosis of C-IRIS have been published [7], although diagnosis remains difficult. To date, there are no clear predictive or diagnostic markers for C-IRIS.

Evidence is accruing against very early commencement of cART after diagnosis of cryptococcal meningitis in HIVinfected patients with three randomized studies demonstrating that deferring cART conferred a survival advantage [8-10]. Death following early initiation of cART has been postulated to be linked to C-IRIS. This is in contrast to evidence from tuberculosis (TB)-HIV coinfection studies in which early cART initiation increased survival in patients with advanced immunodeficiency [11,12], although not in tuberculous meningitis [13].

We sought to define the causes of neurological deterioration post-cART commencement and to identify the clinical and mycological predictors of C-IRIS. In particular, we hypothesized that cryptococcal clearance prior to cART commencement would be associated with improved clinical outcomes.

\section{Methods}

\section{Study design and participants}

Between August 2009 and March 2011, we consecutively enrolled 130 cART-naive, HIV-infected patients experiencing their first episode of cryptococcal meningitis into a prospective, longitudinal cohort study in Durban, South Africa. Patients were 18 years of age or older with a positive cryptococcal antigen $(\mathrm{CrAg})$ or India ink test on cerebrospinal fluid (CSF). Written informed consent was given by the patients or their next-of-kin. Ethics approval was granted by the Biomedical Research and Ethics Committee of University of KwaZulu Natal (BF053/09), Monash University (2009001224), and University of Western Australia (RA/4/1/2541).

\section{Antifungal therapy and combination antiretroviral therapy}

Patients were jointly managed by the local treating team and a single study physician who supervised the management of all patients. Each patient was treated with induction therapy using intravenous amphotericin $(1 \mathrm{mg} / \mathrm{kg})$ for 14 days and therapeutic lumbar punctures were performed as clinically indicated, as per local guidelines [14]. Patients then received 8-12 weeks of consolidation therapy using fluconazole $400 \mathrm{mg}$ followed by $200 \mathrm{mg}$ as maintenance therapy, with a 50\% dose increase for patients receiving rifampicin concurrently. Flucytosine (5-FC) was not available. Standard cART was commenced based on clinical judgment: improvement in conscious state; no further headaches, neck stiffness, confusion, seizures, or vomiting; and improvement in opening pressure (wherever possible, aiming for $<25 \mathrm{cmH}_{2} \mathrm{O}$ ), improvement in renal function, and patient-readiness to initiate cART.

Just prior to cART commencement a lumbar puncture was performed (termed 'clearance lumbar puncture'). The cART regimen was composed of stavudine/ lamivudine/efavirenz until April 2010 when stavudine 
was replaced with tenofovir in the national guidelines [15]. Patients were prospectively followed at a minimum interval of 4 weeks, for 24 weeks from the time of cART commencement. Patients were also systematically evaluated at each clinical visit and data recorded on case report forms, specifically designed to capture data on neurological deterioration. Phone and short-messaging services were utilized to enhance follow-up.

\section{Classification of neurological deterioration events}

Patients were educated thoroughly on the natural history of cryptococcal meningitis and HIV and were informed of the possibility of further neurological deterioration and the need for urgent assessment should this arise. The study physician was available by phone $24 \mathrm{~h}$ a day, 7 days a week for the duration of the study, and patients and families were encouraged to contact the physician if necessary. Symptoms and signs suggestive of neurological deterioration such as worsening headaches, seizures, confusion, neck stiffness, visual change, or limb weakness triggered consideration of a neurological deterioration event. All events were investigated and managed by the study physician in association with local treating teams.

Details of each neurological deterioration episode was retrospectively analyzed by an end-point review committee consisting of the study physician and three experienced HIV physicians and classified as probableC-IRIS, possible-C-IRIS, neurological deteriorationnot C-IRIS, or indeterminate, based on predefined criteria [7]. Patients with a clear alternative explanation for their neurological deterioration or noncompliance to antifungal therapy with a clear increase in cryptococcal CSF load consistent with cryptococcal persistence/ relapse were classified as neurological deterioration-not C-IRIS. Patients with inadequate information (e.g. sudden death at home) were classified as indeterminate. Definitions of possible-C-IRIS and probable-C-IRIS differed only in the strength of the evidence for C-IRIS and both groups were therefore classified as C-IRIS for analyses of predictors of C-IRIS. Patients who did not experience any neurological deterioration were classified as no neurological deterioration.

\section{Examination of cerebrospinal fluid samples and classification by clearance lumbar puncture}

All CSF samples underwent routine analysis for cell count, Gram stain, and India ink testing and were cultured for 30 days. In addition, CSF samples collected at cryptococcal meningitis diagnosis, at clearance lumbar puncture and at neurological deterioration underwent quantitative cryptococcal culture (QnCC). In brief, $100 \mu \mathrm{l}$ of undiluted CSF was cultured, followed by 10-fold serial dilution on five further plates. Remaining CSF was centrifuged and sediment plated on a qualitative plate. CSF and serum CrAg was quantified by latex agglutination (CALAS; Meridian Bioscience, Cincinnati,
Ohio, USA) to a maximum titer of $1: 1024$. All patients had a clearance lumbar puncture performed. On the basis of the clearance lumbar puncture, patients were classified as 'culture negative', if their qualitative cultures were negative for cryptococcal growth at 30 days, and 'culture positive' if their cultures were positive. This classification was blinded to the treating clinicians.

\section{Statistical analysis}

Categorical variables were summarized using frequency and percentages and were compared using a chi-square test or a Fisher's exact test wherever appropriate. Continuous variables were assessed for skew and summarized using mean and standard error or median and interquartile range as appropriate and analyzed using either a $t$-test or a Wilcoxon rank-sum test. Predictors of time to C-IRIS were analyzed using univariable and multivariable Cox proportional-hazards regression. The number of C-IRIS events limited multivariable modeling to three concurrent predictors in any one model. Hazard proportionality was assessed through the analysis of scaled Schoenfeld residuals.

Predictors of culture negativity in the clearance CSF sample were analyzed using logistic regression. Goodnessof-fit of logistic models was assessed using a Hosmer and Lemeshow test. Clinical outcomes of CSF culture negativity were determined by survival analysis. All reported $P$-values are two-tailed and in all analyses $P<0.05$ was considered significant. All analyses were performed using Stata v.12 (StataCorp, College Station, Texas, USA) and GraphPad Prism v5 (La Jolla, California, USA). The funding source took no part in the design or analysis of the study.

\section{Results}

\section{Patient recruitment and demographics}

One hundred and thirty patients were enrolled (Fig. 1); one was erroneously enrolled (HIV-seronegative) and another commenced and ceased cART outside of protocol. Of the remaining 128 patients, three were lost to follow-up (declined to return for cART) and 19 (14.8\%) died during induction antifungal therapy before initiating cART. One hundred and six patients started cART at a median time of 18 days (interquartile range 15-22) after cryptococcal meningitis diagnosis. Post-cART follow-up accrued to 1803 patient-weeks. The median age was 33.5 years; median $\mathrm{CD}^{+}$T-cell count was 35 cells $/ \mu$ l and HIV plasma viral load was $5.2 \log _{10}$ copies $/ \mathrm{ml}$. All patients were positive for CSF CrAg and 118 (92.2\%) had CSF cultureconfirmed cryptococcal infection.

\section{Neurological deterioration and survival}

Forty-three patients had neurological deterioration events $(n=27,25.5 \%$ C-IRIS; and $n=16,15.1 \%$ 


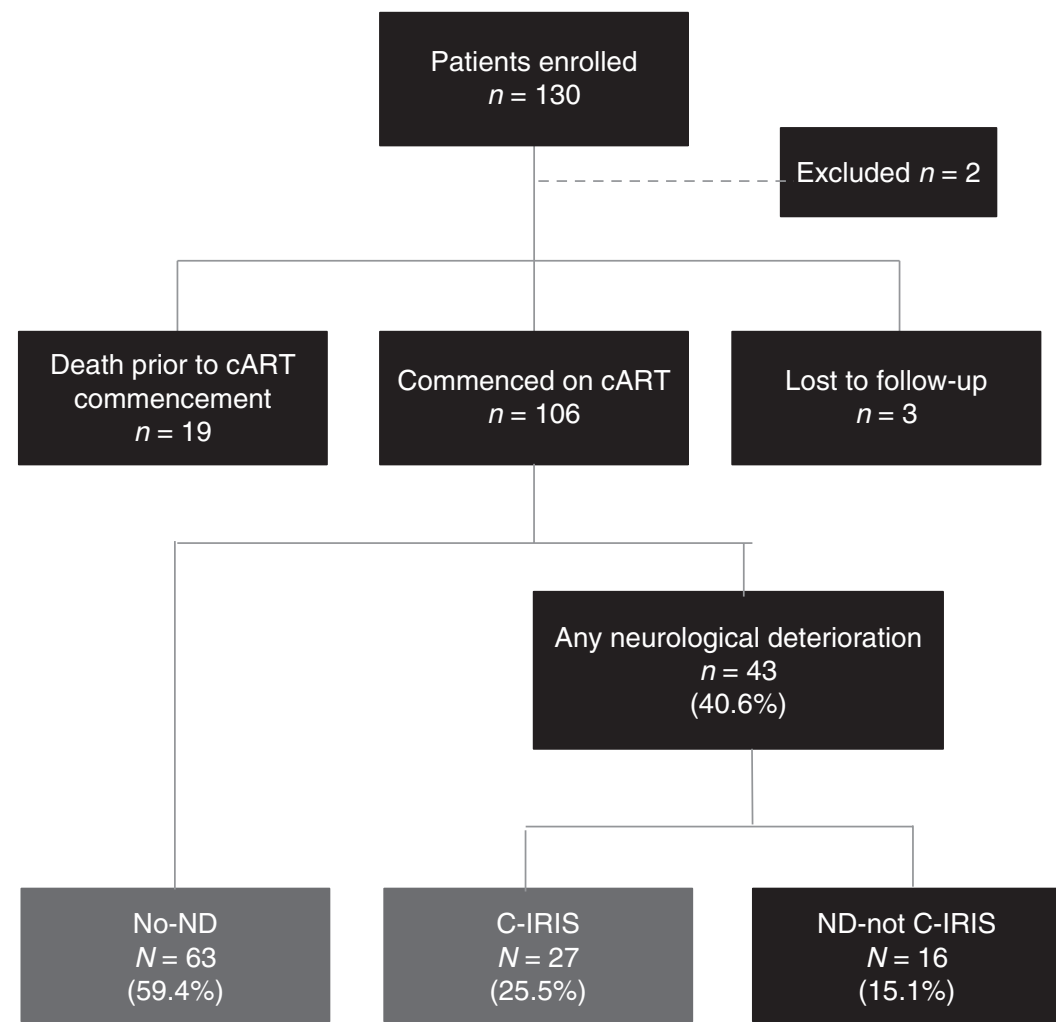

Fig. 1. Patient recruitment and disposition flowchart. CART, combination antiretroviral therapy; C-IRIS, cryptococcosisassociated immune reconstitution inflammatory syndrome; ND, neurological deterioration.

neurological deterioration-not C-IRIS), whereas 63 had no neurological deterioration (59.4\%; Fig. 1). Eight patients experienced multiple neurological deterioration events. Clinical features of neurological deterioration included headache, nausea/vomiting, neck pain, and confusion, which occurred in $74.6,58.2,38.2$, and $21.8 \%$, respectively. The neurological deterioration events $(n=55)$ were classified as C-IRIS $(n=34$; $61.8 \%)$, neurological deterioration-not C-IRIS $(n=14$; $25.5 \%)$, and indeterminate $(n=7 ; 12.7 \%)$. The causes of neurological deterioration-not C-IRIS were cryptococcal persistence/relapse $(n=6)$, shunt-related problems $(n=2)$, renal failure $(n=2)$, pulmonary $\mathrm{TB}(n=2)$, varicella-zoster infection $(n=1)$, and migraine $(n=1)$. Of the seven indeterminate events, three died in hospital without sufficient investigations and four died out of hospital. Twenty-one (19.8\%) deaths were recorded after cART commencement. Survival at 6 months post-cART was $80.2 \%$ in the cohort who commenced cART.

\section{Predictors of cryptococcosis-associated immune reconstitution inflammatory syndrome}

The C-IRIS group, when compared with the no neurological deterioration group, had lower $\mathrm{CD} 4^{+}$ T-cell counts pre-cART (median 16 vs. 36 cells/ $\mu$, $P=0.015)$, but there was no significant difference in age, sex, HIV viral load, or BMI between groups (Table 1). Seizures at cryptococcal meningitis presentation were more common in the C-IRIS group $(P=0.028)$.
On the basis of the first CSF sample collected at cryptococcal meningitis diagnosis, the C-IRIS group compared to the no neurological deterioration group, had a lower CSF protein level (median 0.70 vs. $0.91 \mathrm{~g} / 1$, $P=0.013$ ); lower CSF neutrophil count (median 0 vs. 6 cells $/ \mu 1, P=0.018$ ); lower CSF lymphocyte count (median 10 vs. 34 cells/ $\mu$ l, $P=0.006$ ); and higher CSF cryptococcal burden as measured by QnCC (median 111000 vs. 1800 colony forming unit $(\mathrm{CFU}) / \mathrm{ml}$, $P=0.004 ;$ Table 1).

When we assessed the clearance lumbar puncture (median time 15 days from cryptococcal meningitis diagnosis), fewer patients in the C-IRIS group compared to the no neurological deterioration group were culture negative (25.9 vs. $61.9 \%, P=0.002)$; and the C-IRIS group had lower CSF protein levels and total leucocyte counts (median 0.57 vs. $0.94 \mathrm{~g} / 1, P=0.003$ and 18 vs. 39 , $P=0.045$, respectively; Table 1). QnCCs for clearance lumbar puncture were available for $97.2 \%$ of patients and were not significantly different between groups. Lumbar puncture opening pressure, CSF glucose, and CSF and serum $\mathrm{CrAg}$ were not significantly different between groups.

$\mathrm{CD} 4{ }^{+}$T-cell counts remained lower in the C-IRIS group compared to the no neurological deterioration group at week $04(P=0.0042)$ but were similar by week 12 , whereas there were no differences in HIV viral load 


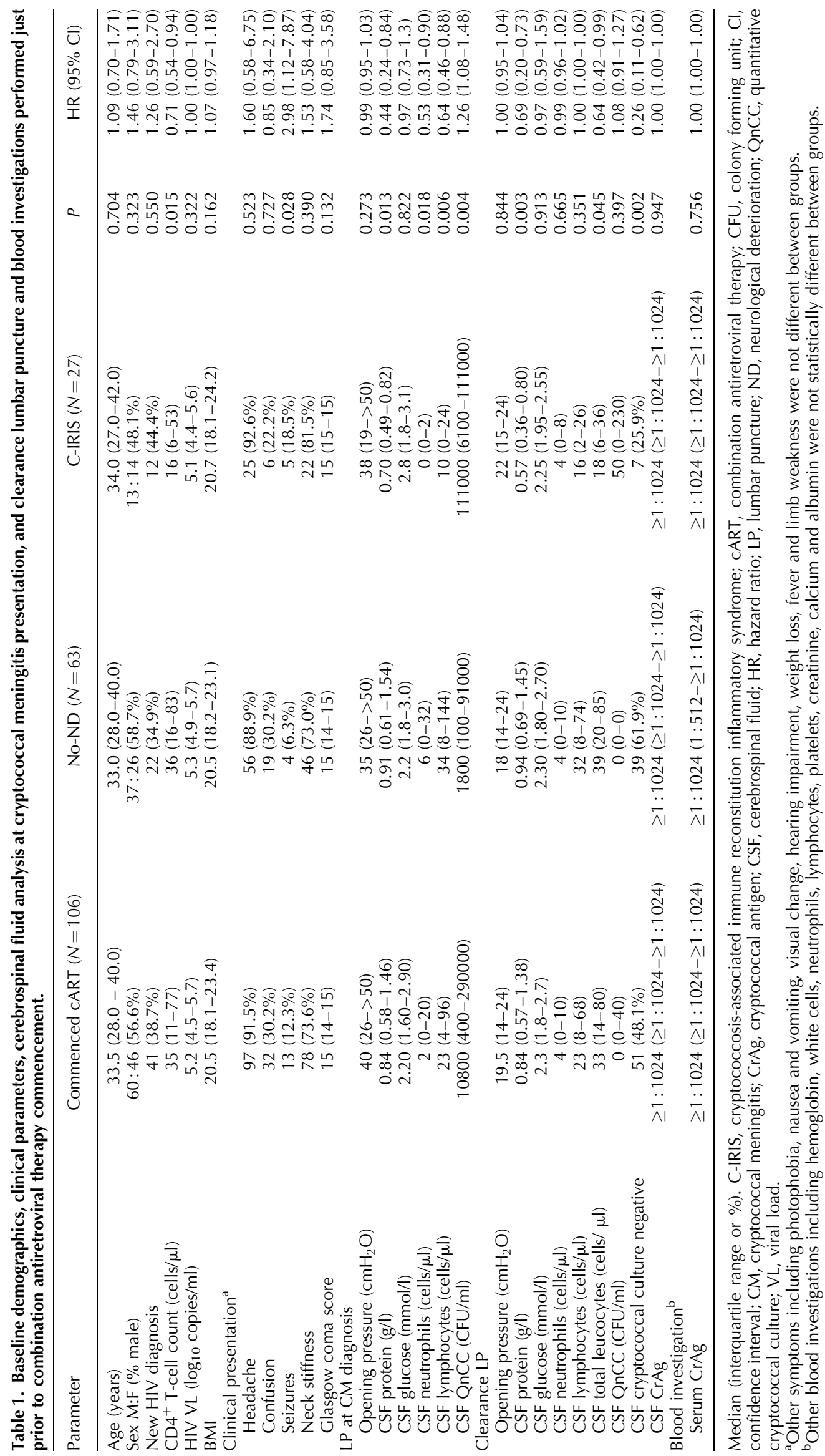


Table 2. Three-parameter multivariate analysis for cryptococcosisassociated immune reconstitution inflammatory syndrome.

\begin{tabular}{lccc}
\hline Parameter & $P$ & $\mathrm{HR}$ & $95 \% \mathrm{Cl}$ \\
\hline Clearance LP - culture negative & 0.010 & 0.2691 & $0.0986-0.7342$ \\
CD4 ${ }^{+}$T cell over 24 weeks & 0.026 & 0.9895 & $0.9804-0.9987$ \\
Clearance LP - CSF protein & 0.059 & 0.5282 & $0.2726-1.0233$ \\
\hline
\end{tabular}

$\mathrm{Cl}$, confidence interval; $\mathrm{CSF}$, cerebrospinal fluid; $\mathrm{HR}$, hazard ratio; $\mathrm{LP}$, lumbar puncture.

(supplementary Figure 1, http://links.lww.com/QAD/ A364). Analyzing time-varying $\mathrm{CD} 4^{+} \mathrm{T}$-cell counts over 24 weeks demonstrated that every $100 \mathrm{CD}^{+}$T-cell increase on cART was associated with a $62 \%$ reduction in the rate of C-IRIS [hazard ratio (HR) 0.28, 95\% confidence interval (CI) $0.13-0.59, P=0.001]$. Changes in serum CrAg level over 24 weeks were not associated with C-IRIS $(P=0.324)$.

Using multivariable analysis, a negative CSF cryptococcal culture at cART initiation and increasing $\mathrm{CD} 4^{+} \mathrm{T}$ cells over 24 weeks of cART were both independently associated with decreased rates of C-IRIS $(P=0.010$, HR 0.27 and $P=0.026$, HR 0.99 , respectively), whereas lower CSF protein on the clearance lumbar puncture showed a trend toward an association with decreased rates of C-IRIS $(P=0.059$, HR 0.53) (Table 2).

\section{Predictors of cerebrospinal fluid cryptococcal culture negativity and its clinical outcomes}

On the basis of the clearance lumbar puncture results available for all 106 patients, patients were divided into 'culture-negative' $(n=51,48.1 \%)$ or 'culture-positive' $(n=55,51.9 \%)$ groups. The number of amphotericin doses (median 14 in both groups) and duration of antifungal therapy prior to the clearance lumbar puncture (14 vs. 15 days) and time to starting cART following cryptococcal meningitis diagnosis (17 vs. 20 days) were similar between groups $(P=0.332,0.255$, and 0.180 , respectively) (Table 3 ).

The culture-negative compared to the culture-positive group had higher pre-cART $\mathrm{CD}^{+}{ }^{+}$T-cell counts (median 42 vs. 33 cells $/ \mu l, P=0.009$ ), higher neutrophil counts in CSF at cryptococcal meningitis presentation (median 6 vs. 0 cells $/ \mu l, \quad P=0.031$ ); and higher lymphocyte and total leucocyte counts in CSF from the clearance lumbar puncture (median 34 vs. 16 cells $/ \mu 1$, $P=0.006$ and 54 vs. 28 cells $/ \mu l, P=0.012$, respectively) (Table 3). Seizures as a presenting feature were less common in the culture-negative group (3.6 vs. $20.0 \%$, $P=0.023)$. CSF QnCC performed on the first available $\mathrm{CSF}$ and the clearance lumbar puncture were both lower in the culture-negative compared to the culture-positive group $(P=<0.001$ and 0.006 , respectively) as were the CSF and serum CrAg titers $(P=0.015$ and 0.002 , respectively) (Table 3 ).
Using multivariable analysis, lower QnCC in CSF from the clearance lumbar puncture and serum $\mathrm{CrAg}$ were independent predictors of CSF culture negativity $(P=0.007$, odds ratio $0.063,95 \%$ CI $0.008-0.468$ and $P=0.025$, odds ratio $0.998,95 \%$ CI $0.996-1.000$, respectively). CSF lymphocyte count and blood $\mathrm{CD}^{+}$ T-cell count contributed to the model $(P=0.408$ and 0.610 , respectively) (Table 3 ).

To test the hypothesis that CSF cryptococcal clearance prior to cART commencement is associated with improved clinical outcomes, we specifically compared neurological deterioration, C-IRIS, cryptococcal relapse/persistence and death between these two groups. Using survival analysis, the culture-negative group compared to the culture-positive group had significantly reduced rates of neurological deterioration (23.5 vs. $56.4 \%$, HR $0.33,95 \%$ CI $0.18-0.60, P=0.0003$ ) and CIRIS (13.7 vs. $36.4 \%$, HR 0.33 , 95\% CI $0.15-0.70$, $P=0.0042)$. All neurological deterioration caused by cryptococcal relapse/persistence $(n=11)$ occurred in the culture-positive group (HR $0.12,95 \%$ CI $0.04-0.38$, $P=0.0004$ ) (Fig. 2). There were fewer deaths in the culture-negative group but the difference was not statistically significant (13.7 vs. $25.5 \%$, HR $0.52,95 \%$ CI $0.22-1.22, P=0.13$ ) (Fig. 2).

\section{Discussion}

Persistent CSF cryptococcal growth at initiation of cART and advanced immunodeficiency are independent predictors of C-IRIS. Importantly, patients who cleared cryptococcus from their CSF prior to commencing cART not only experienced significantly lower rates of C-IRIS, but also had fewer episodes of neurological deterioration and cryptococcal relapse/persistence, suggesting that cryptococcal clearance prior to initiation of cART is a significant determinant of clinical outcome in HIV-infected patients with cryptococcal meningitis, as supported by a recent review [16]. Previous studies show slower rates of cryptococcal clearance are associated with increased early mortality [17].

We demonstrated that a lower blood CD $4^{+}$T-cell count, CSF protein, lymphocyte and neutrophil count, and, in particular, a higher cryptococcal burden are associated with an increased risk of developing C-IRIS. These findings confirm previously identified predictors of C-IRIS including a lower baseline $\mathrm{CD}^{+}{ }^{+}$T-cell count $[18,19]$ and reduced CSF inflammation [20]. Others have shown that a higher CSF CrAg and lower CSF white cell count to be associated with worsened cryptococcal meningitis outcomes [21]. We show gains in $\mathrm{CD} 4^{+} \mathrm{T}$-cell count as protective for C-IRIS highlighting immune competence as beneficial. This suggests the immunopathology underlying C-IRIS may not reflect the 


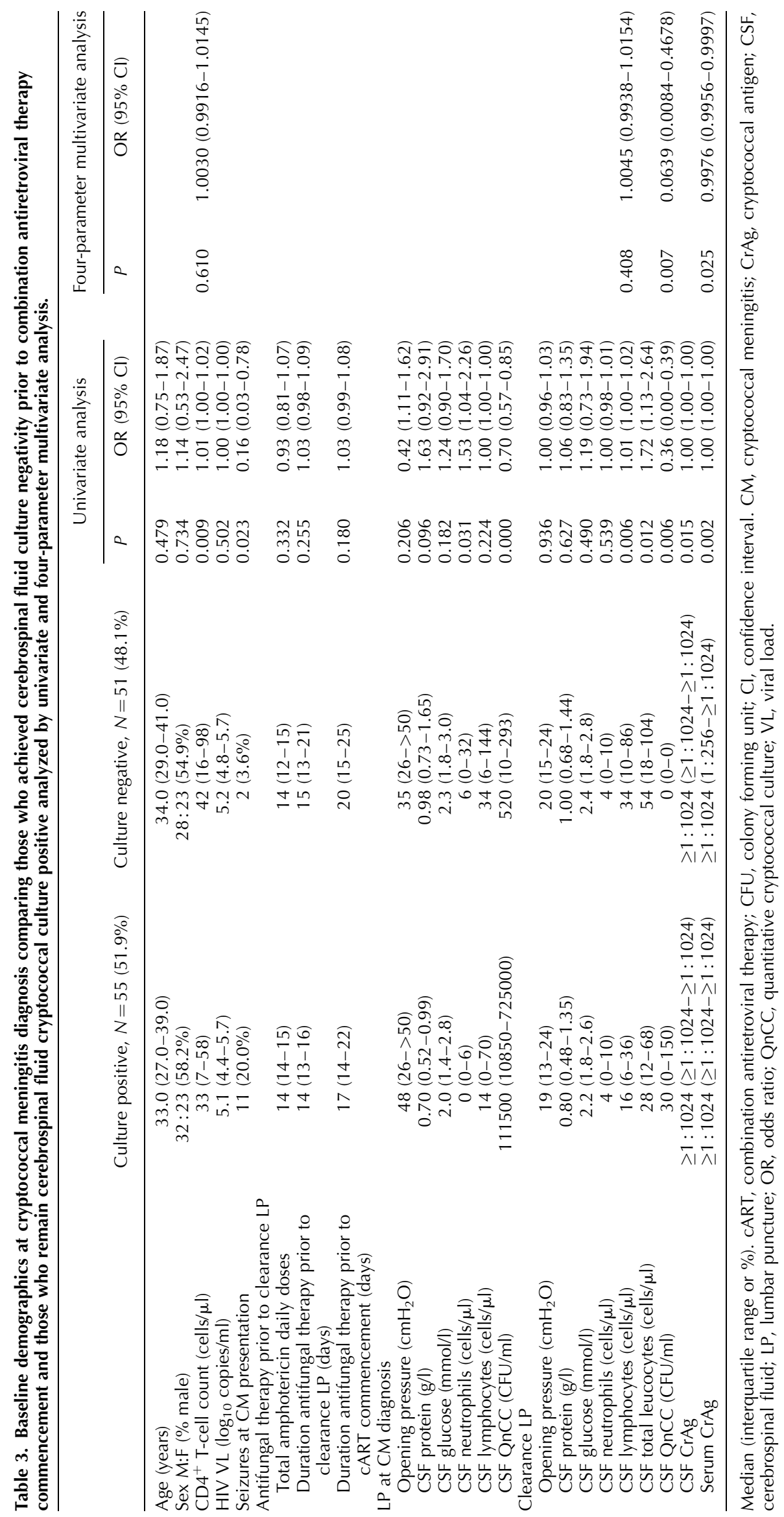




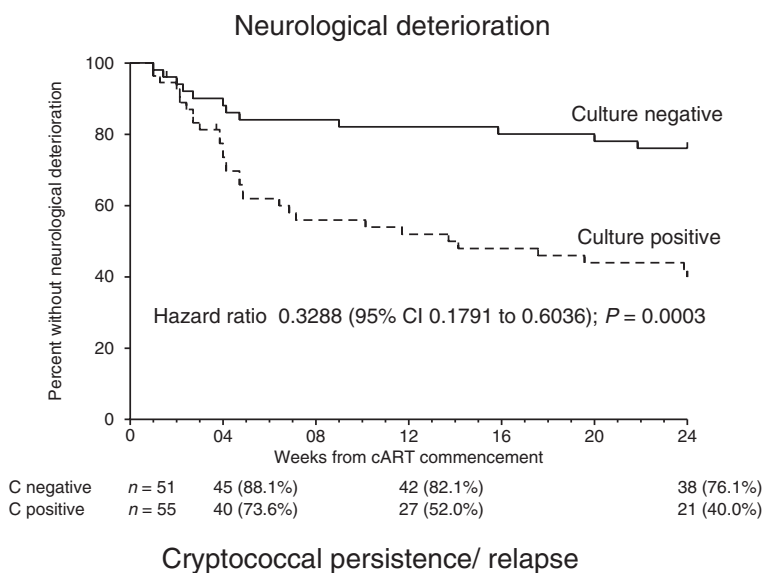

Cryptococcal persistence/ relapse

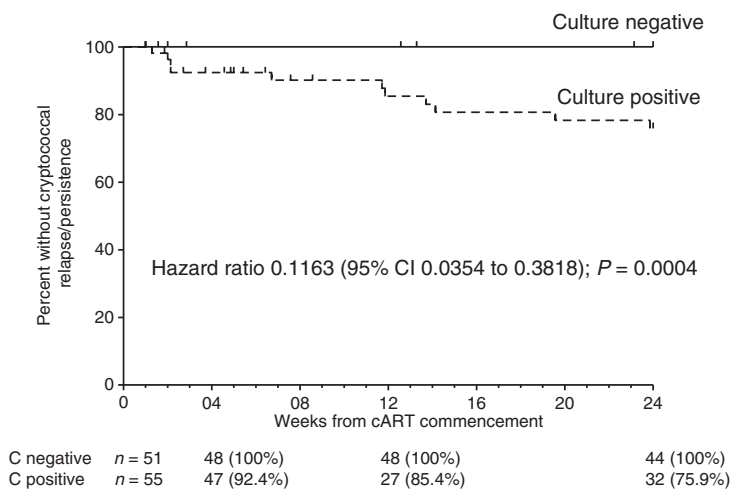

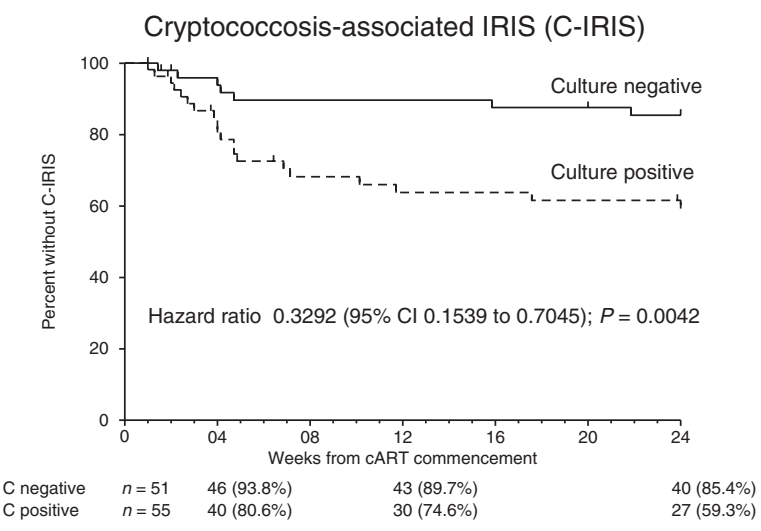

Deaths

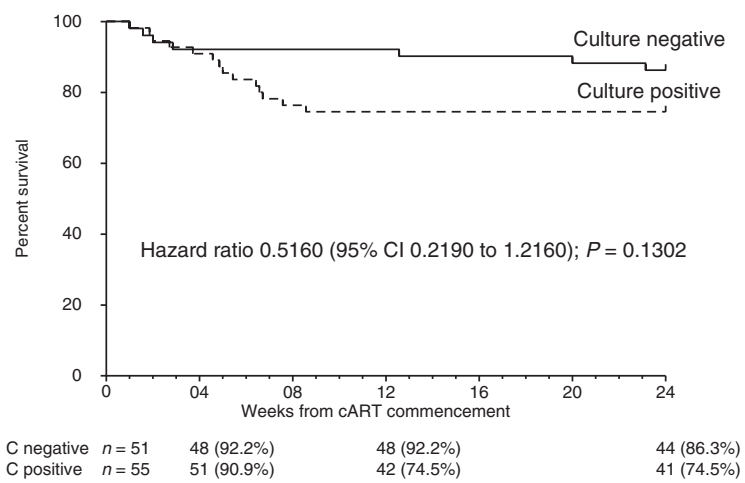

Fig. 2. Kaplan-Meier curves on the impact of cerebrospinal fluid (CSF) cryptococcal culture negativity pre-combination antiretroviral therapy (CART) on neurological deterioration (ND), cryptococcosis-associated immune reconstitution inflammatory syndrome (C-IRIS), cryptococcal relapse/persistence and mortality. Solid line (culture negative) and broken line (culture positive). Patients who were able to achieve CSF cryptococcal culture negativity prior to cART commencement had significantly lower episodes of ND, C-IRIS, and cryptococcal relapse/persistence compared to those who remained cryptococcal culture positive. Survival was improved in the culture-negative group but this was not statistically significant. $\mathrm{Cl}$, confidence interval; $\mathrm{HR}$, hazard ratio.

numerical gain of $\mathrm{CD} 4^{+} \mathrm{T}$ cells but rather the restoration of a dysfunctional immune response.

Further, to our knowledge, we demonstrate for the first time that a persistent high cryptococcal burden in the CSF prior to initiation of cART is also a risk factor for C-IRIS. Despite a higher fungal burden, the more immunodeficient C-IRIS group seemed less able to mount an adequate cellular response, possibly explained by the immunosuppressive effects of the polysaccharide capsule [22]. Early reports of cryptococcal meningitis demonstrated an acellular CSF profile to be associated with early death and treatment failure [23]; we show this is also associated with an increased risk for C-IRIS.

Our finding that a gain in $\mathrm{CD} 4^{+} \mathrm{T}$ cells is protective for C-IRIS may seem counterintuitive and is in contrast to a previous study in C-IRIS often quoted as evidence for a rapid rise in $\mathrm{CD} 4^{+} \mathrm{T}$ cells as a risk factor for IRIS [24]. However, this study reported the absolute difference in $\mathrm{CD} 4^{+} \mathrm{T}$ cells from baseline to 6 months and four of the 11 patients in their C-IRIS group died within 6 months; hence, the small remaining number of patients may have skewed the findings of the study. In contrast, our study measured $\mathrm{CD} 4^{+}$T-cell counts repeatedly at weeks 4,12 , and 24 in a much larger number of patients and used a time-to-event analysis. We have demonstrated in this cohort of patients that C-IRIS is not associated with an increase in circulating $\mathrm{CD} 4^{+} \mathrm{T}$ cells reactive with cryptococcal mannoprotein [25]. Although, compartmentalization of T-cell responses to the CNS is possible, a role for innate immune responses should be considered, as suggested in TB-IRIS [26,27].

CSF protein is usually elevated in patients with cryptococcal meningitis and lower levels have been associated with higher cryptococcal meningitis early mortality [28]. Patients with C-IRIS had a significantly lower CSF protein at cryptococcal meningitis presentation than the no neurological deterioration group, as previously reported [20], and this difference was further exaggerated during induction antifungal therapy. An explanation for this is not immediately apparent. CSF proteins are derived from passage of proteins across the blood-brain barrier; from inflammatory cells [22], damage to and necrosis of the meninges and neuronal 
cells [22] and from cryptococcal cell wall breakdown. Exploratory proteomic analysis may be instructive as more than 2630 proteins have been found in normal CSF [29].

Early cryptococcal clearance has been used in treatment studies as a measure of mycological success [30-32] but to date, this has not been accepted as a surrogate marker of clinical success. We clearly show that achieving a negative CSF culture prior to starting cART conferred significantly improved clinical outcomes. The relatively low number of deaths in our study group may not have conferred adequate power to demonstrate a statistically significant difference in mortality during the defined follow-up period. Cryptococcal meningitis management guidelines [14,33,34] in HIV-infected patients offer a rank order of recommended antifungal agents with varying durations, based predominantly on fungal clearance efficacy. Studies have shown that early mortality in cryptococcal meningitis correlates strongly with the baseline CSF QnCC [31]. We show that in patients receiving similar doses of amphotericin, those with a higher $\mathrm{CD}^{+}{ }^{+} \mathrm{T}$-cell count, lower QnCC, lower CSF and serum CrAg, and a more cellular CSF are more likely to achieve CSF culture negativity. We propose that patients could be potentially stratified by fungal burden at cryptococcal meningitis diagnosis, degree of immunodeficiency and CSF profile into two-tier or three-tier risk strata with the goal of achieving CSF culture negativity, prior to cART commencement. Those with poor predictive markers could be targeted for enhanced antifungal therapy or adjunctive therapy including interferon- $\gamma$ therapy [35].

No patients in the culture-negative group developed cryptococcal persistence/relapse, suggesting that empirical re-induction therapy in this group is not always necessary. QnCCs, although more labor-intensive, may serve as a baseline measure of fungal burden to be compared to future neurological deterioration events; an increase in QnCC compared to baseline may suggest cryptococcal relapse necessitating intensification of antifungal therapy.

Timing of cART initiation in the presence of concurrent opportunistic infections is contentious. In cryptococcal meningitis-HIV co-infection, one small randomized controlled study (RCT) showed excess mortality in the early arm compared to the delayed arm ( $\leq 72-\mathrm{h}$ vs. $\geq 10$ weeks after cryptococcal meningitis diagnosis) [10], whereas a smaller RCT demonstrated higher C-IRIS in the early arm vs. the delayed arm (within 7-10 days vs. after 28 days of antifungal therapy commencement) [8]. Fluconazole was used as induction therapy in the former study and limited therapeutic lumbar punctures were performed [10], two important factors that differed from our study. In the latter study, $0.7 \mathrm{mg} / \mathrm{kg}$ of amphotericin was used [8] and the authors previously reported the benefits of inpatient cART commencement in reducing patient mortality in HIV-cryptococcal meningitis-TB coinfected men [36]. The large 'cryptococcal meningitis optimal ART timing' study was terminated at 177 persons (35\% of target enrolment), for reasons of unacceptable mortality in the early arm compared to the deferred arm (day 9-13 vs. $\geq 5$ weeks after cryptococcal meningitis diagnosis) [9]. Patients with low CSF white blood cell counts and altered mental state, in particular, did better in the deferred arm [9]. We speculate patients in early arms of these three studies may have had larger residual fungal burden at cART initiation. Adjunctive management strategies such as intracranial pressure management may not have been optimally addressed.

Other prospective non-randomized cryptococcal meningitis studies commenced cART at a median of 5 weeks (range 1-8) [37] to as late as after 7 weeks [38] after cryptococcal meningitis diagnosis. We commenced cART at a median time of 18 days after cryptococcal meningitis diagnosis and achieved a remarkable overall survival of $80 \%$ at 24 weeks post-cART initiation (higher than either of the cryptococcal meningitis optimal ART timing study arms). Delaying cART to conform to current RCT findings in resource-limited settings may risk further comorbidities, hospital-acquired infections, unmonitored deterioration, losses to follow-up and death as illustrated by a $29 \%$ loss between completion of induction therapy and cART initiation in a Ugandan study [28].

We acknowledge that our study has limitations. Until a firm diagnostic marker for C-IRIS is developed, studies of C-IRIS are open to criticism. Our experienced endpoint review committee actively excluded indeterminate neurological deterioration events and the 100\% followup rate enhanced the validity of our comparator no neurological deterioration group. We focused solely on the central nervous system manifestations and may have underestimated the true incidence of C-IRIS. We had limited histopathological confirmation.

In summary, persistent cryptococcal burden and advanced immunodeficiency prior to commencing cART may drive aberrant immune responses against Cryptococci leading to C-IRIS. Our study demonstrated that CSF cryptococcal clearance prior to initiation of cART is associated with improved clinical outcomes. Where assessing this is not possible, a combination of markers (lower CD $4^{+}$T-cell count, higher CSF and serum CrAg, and lower CSF neutrophil and lymphocyte counts precART) might be used to predict a higher cryptococcal burden. The benefits of attaining CSF cryptococcal culture negativity prior to cART commencement should be further evaluated in future clinical studies.

\section{Acknowledgements}

The authors would like to acknowledge the patients and their families, the medical, nursing, laboratory and 
support staff at King Edward VIII Hospital, Durban and Department of Microbiology, Inkosi Albert Luthuli Central Hospital, Durban. This work was supported by REACH Initiative (Research and Education in HIV/ AIDS for Resource Poor Countries).

Authors' contribution: M.A.F., C.C.C., S.R.L., M.Y.S.M. and J.H.E. designed the study; C.C.C., M.A.F., S.R.L., T.S. and V.N. analyzed the data and wrote the manuscript. C.C.C., B.I.G. and S.O. conducted the clinical study; M.A.F., C.C.C., J.H.E., M.-Y.S.M. formed the end-point review committee. A.A.D. performed the cryptococcal laboratory work under the guidance of Y.C. and C.C.C. Authors M.A.F., S.R.L., C.C.C., J.H.E., T.N. and M.-Y.S.M. were members of the protocol steering committee. All read and approved the final manuscript.

C.C.C. was supported by the Australian Commonwealth Government for the Australian Postgraduate Award 2009 and Australian NHMRC (National Health and Medical Research Council) Postgraduate Scholarship 2010-2012. T.N. was supported in part by an international Early Career Scientist grant from the Howard Hughes Medical Institute and by the South African Department of Science and Technology/National Research Foundation Research Chairs Initiative. S.R.L. is an NHMRC practitioner fellow. M.A.F. was supported by NHMRC grant 510448.

\section{Conflicts of interest}

All authors have no commercial or other association that might pose a conflict of interest.

Findings in this manuscript have in part been presented at the 8th International Conference of Cryptococcus and Cryptococcosis (Charleston, 2011), Australasian HIV/AIDS conference (Canberra, 2011), Conference on Retroviruses and Opportunistic Infections (Seattle, 2012) and World AIDS Conference (Washington, DC, 2012).

\section{References}

1. Park BJ, Wannemuehler KA, Marston BJ, Govender N, Pappas PG, Chiller TM. Estimation of the current global burden of cryptococcal meningitis among persons living with HIV/AIDS. AIDS 2009; 23:525-530.

2. French MA. Immune reconstitution inflammatory syndrome: immune restoration disease 20 years on. Med J Aust 2012; 196:318-321.

3. Muller M, Wandel S, Colebunders R, Attia S, Furrer H, Egger M. Immune reconstitution inflammatory syndrome in patients starting antiretroviral therapy for HIV infection: a systematic review and meta-analysis. Lancet Infect Dis 2010; 10:251-261.

4. French MA, Price $P$, Stone SF. Immune restoration disease after antiretroviral therapy. AIDS 2004; 18:1615-1627.

5. Robertson J, Meier M, Wall J, Ying J, Fichtenbaum CJ. Immune reconstitution syndrome in HIV: validating a case definition and identifying clinical predictors in persons initiating antiretroviral therapy. Clin Infect Dis 2006; 42:1639-1646.
6. Shelburne SA III, Hamill RJ, Rodriguez-Barradas MC, Greenberg $\mathrm{SB}$, Atmar RL, Musher DW, et al. Immune reconstitution inflammatory syndrome: emergence of a unique syndrome during highly active antiretroviral therapy. Medicine (Baltimore) 2002; 81:213-227.

7. Haddow LJ, Colebunders R, Meintjes G, Lawn SD, Elliott JH, Manabe $Y C$, et al. Cryptococcal immune reconstitution inflammatory syndrome in HIV-1-infected individuals: proposed clinical case definitions. Lancet Infect Dis 2010; 10: 791-802.

8. Bisson GP, Molefi M, Bellamy S, Thakur R, Steenhoff A, Tamuhla $N$, et al. Early versus delayed antiretroviral therapy and cerebrospinal fluid fungal clearance in adults with HIV and cryptococcal meningitis. Clin Infect Dis 2013; 56:11651173.

9. Boulware DR, Meya DB, Muzoora C, Rolfes MA, Huppler Hullsiek K, Musubire A, et al. ART initiation within the first 2 weeks of cryptococcal meningitis is associated with higher mortality: a multisite randomized study. Conference on Retroviruses and Opportunistic Infections. Atlanta, Georgia 2013.

10. Makadzange AT, Ndhlovu CE, Takarinda K, Reid M, Kurangwa $M$, Gona $P$, et al. Early versus delayed initiation of antiretroviral therapy for concurrent HIV infection and cryptococcal meningitis in sub-Saharan Africa. Clin Infect Dis 2010; 50:1532-1538.

11. Abdool Karim SS, Naidoo K, Grobler A, Padayatchi N, Baxter C, Gray $A L$, et al. Integration of antiretroviral therapy with tuberculosis treatment. $N$ Eng/ / Med 2011; 365:1492-1501.

12. Blanc FX, Sok T, Laureillard D, Borand L, Rekacewicz C, Nerrienet $E$, et al. Earlier versus later start of antiretroviral therapy in HIV-infected adults with tuberculosis. N Eng/ / Med 2011; 365:1471-1481.

13. Torok ME, Yen NT, Chau TT, Mai NT, Phu NH, Mai PP et al. Timing of initiation of antiretroviral therapy in human immunodeficiency virus (HIV)-associated tuberculous meningitis. Clin Infect Dis 2011; 52:1374-1383.

14. McCarthy K, Meintjes G. Guidelines for the prevention, diagnosis and management of cryptococcal meningitis and disseminated cryptococcosis in HIV-infected patients. $S$ Afr J HIV Med 2007; 8 (Spring):25-35.

15. National Department of Health SA. Clinical guidelines for the management of HIV \& AIDS in adults and adolescents. National Department of Health SA; 2010, http://www.fidssa.co.za/ Guidelines/2010_Adult_ART_Guidelines.pdf. [Accessed 10 June 2012]

16. Wiesner DL, Boulware DR. Cryptococcus-related immune reconstitution inflammatory syndrome (IRIS): pathogenesis and its clinical implications. Curr Fungal Infect Rep 2011; 5:252-261.

17. Bicanic T, Muzoora C, Brouwer AE, Meintjes G, Longley N, Taseera $K$, et al. Independent association between rate of clearance of infection and clinical outcome of HIV-associated cryptococcal meningitis: analysis of a combined cohort of 262 patients. Clin Infect Dis 2009; 49:702-709.

18. French MA, Lenzo N, John M, Mallal SA, McKinnon EJ, James $\mathrm{IR}$, et al. Immune restoration disease after the treatment of immunodeficient HIV-infected patients with highly active antiretroviral therapy. HIV Med 2000; 1:107-115.

19. Lortholary O, Fontanet A, Memain N, Martin A, Sitbon K, Dromer $F$. Incidence and risk factors of immune reconstitution inflammatory syndrome complicating HIV-associated cryptococcosis in France. AIDS 2005; 19:1043-1049.

20. Boulware DR, Bonham SC, Meya DB, Wiesner DL, Park GS, Kambugu $A$, et al. Paucity of initial cerebrospinal fluid inflammation in cryptococcal meningitis is associated with subsequent immune reconstitution inflammatory syndrome. / Infect Dis 2010; 202:962-970.

21. Anekthananon T, Manosuthi $\mathrm{W}$, Chetchotisakd $\mathrm{P}$, Kiertiburanakul S, Supparatpinyo K, Ratanasuwan W, et al. Predictors of poor clinical outcome of cryptococcal meningitis in HIVinfected patients. Int / STD AIDS 2011; 22:665-670.

22. Thompson EJ. Different blood-CSF barriers. Proteins of the cerebrospinal fluid - analysis and interpretation in the diagnosis and treatment of neurological disease. San Diego: Elsevier Ltd; 2005.

23. Diamond RD, Bennett JE. Prognostic factors in cryptococcal meningitis. A study in 111 cases. Ann Intern Med 1974; 80:176181. 
24. Bicanic T, Meintjes G, Rebe K, Williams A, Loyse A, Wood R, et al. Immune reconstitution inflammatory syndrome in HIVassociated cryptococcal meningitis: a prospective study. J Acquir Immune Defic Syndr 2009; 51:130-134.

25. Chang CC, Lim A, Omarjee S, Levitz SM, Gosnell BI, Spelman T, et al. Cryptococcosis-IRIS is associated with lower cryptococcus-specific IFN-gamma responses before antiretroviral therapy but not higher T-cell responses during therapy. I Infect Dis 2013; [Epub ahead of print].

26. Chang CC, Crane M, Zhou J, Mina M, Post J, Cameron B, et al. HIV and co-infections. Immunol Rev 2013; 254:114-142.

27. Oliver BG, Elliott JH, Price P, Phillips M, Saphonn V, Vun MC, et al. Mediators of innate and adaptive immune responses differentially affect immune restoration disease associated with Mycobacterium tuberculosis in HIV patients beginning antiretroviral therapy. J Infect Dis 2010; 202:1728-1737.

28. Kambugu A, Meya DB, Rhein J, O'Brien M, Janoff EN, Ronald $A R$, et al. Outcomes of cryptococcal meningitis in Uganda before and after the availability of highly active antiretroviral therapy. Clin Infect Dis 2008; 46:1694-1701.

29. Schutzer SE, Liu T, Natelson BH, Angel TE, Schepmoes AA Purvine SO, et al. Establishing the proteome of normal human cerebrospinal fluid. PLOS ONE 2010; 5:e10980.

30. Bicanic T, Meintjes G, Wood R, Hayes M, Rebe K, Bekker LG, et al. Fungal burden, early fungicidal activity, and outcome in cryptococcal meningitis in antiretroviral-naive or antiretroviral-experienced patients treated with amphotericin B or fluconazole. Clin Infect Dis 2007; 45:76-80.

31. Brouwer AE, Rajanuwong A, Chierakul W, Griffin GE, Larsen RA, White NJ, et al. Combination antifungal therapies for HIVassociated cryptococcal meningitis: a randomised trial. Lancet 2004; 363:1764-1767.
32. Pappas PG, Chetchotisakd P, Larsen RA, Manosuthi W, Morris $\mathrm{MI}$, Anekthananon T, et al. A phase II randomized trial of amphotericin B alone or combined with fluconazole in the treatment of HIV-associated cryptococcal meningitis. Clin Infect Dis 2009; 48:1775-1783.

33. Perfect JR, Dismukes WE, Dromer F, Goldman DL, Graybill JR, Hamill RJ, et al. Clinical practice guidelines for the management of cryptococcal disease: 2010 update by the Infectious Diseases Society of America. Clin Infect Dis 2010; 50:291-322.

34. WHO. Rapid advice: diagnosis, prevention and management of cryptococcal disease in HIV-infected adults, adolescents and children. WHO library cataloguing-in-publication data; 2011. http://www.who.int/hiv/pub/cryptococcal_disease2011/en/. [Accessed 10 June 2012]

35. Jarvis JN, Meintjes G, Rebe K, Williams GN, Bicanic T, Williams $A$, et al. Adjunctive interferon-gamma immunotherapy for the treatment of HIV-associated cryptococcal meningitis: a randomized controlled trial. AIDS 2012; 26:1105-1113.

36. Bisson GP, Nthobatsong R, Thakur R, Lesetedi G, Vinekar K, Tebas $P$, et al. The use of HAART is associated with decreased risk of death during initial treatment of cryptococcal meningitis in adults in Botswana. J Acquir Immune Defic Syndr 2008; 49:227-229.

37. Longley N, Muzoora C, Taseera K, Mwesigye J, Rwebembera J, Chakera A, et al. Dose response effect of high-dose fluconazole for HIV-associated cryptococcal meningitis in southwestern Uganda. Clin Infect Dis 2008; 47:1556-1561.

38. Sungkanuparph S, Filler SG, Chetchotisakd P, Pappas PG, Nolen TL, Manosuthi W, et al. Cryptococcal immune reconstitution inflammatory syndrome after antiretroviral therapy in AIDS patients with cryptococcal meningitis: a prospective multicenter study. Clin Infect Dis 2009; 49:931-934. 